\title{
Molecular gas in the star-forming region IRAS 08589-4714 ${ }^{\star}$ (Research Note)
}

\author{
Hugo P. Saldaño ${ }^{12}$, J. Vásquez ${ }^{234}$, C. E. Cappa ${ }^{234}$, M. Gómez ${ }^{12}$, N. Duronea ${ }^{23}$, and M. Rubio ${ }^{5}$ \\ 1 Observatorio Astronómico, Universidad Nacional de Córdoba, 5000 Córdoba, Argentina \\ e-mail: hugosaldanio@oac.uncor.edu \\ 2 CONICET, Consejo Nacional de Investigaciones Científicas y Técnicas, Argentina \\ 3 Instituto Argentino de Radioastronomía, CONICET, CCT La PLata, C.C.5, 1894 Villa Elisa, Argentina \\ 4 Facultad de Ciencias Astronómicas y Geofísicas, Universidad Nacional de la Plata, Paseo del Bosque s/n, 1900 La Plata, Argentina \\ 5 Departamento de Astronomía, Universidad de Chile, Casilla 36, Santiago de Chile, Chile
}

Received 7 December 2015 / Accepted 13 July 2016

\begin{abstract}
Aims. We present an analysis of the region IRAS 08589-4714 with the aim of characterizing the molecular environment. Methods. We observed the ${ }^{12} \mathrm{CO}(3-2),{ }^{13} \mathrm{CO}(3-2), \mathrm{C}^{18} \mathrm{O}(3-2), \mathrm{HCO}^{+}(3-2)$, and $\mathrm{HCN}(3-2)$ molecular lines in a region of $150^{\prime \prime} \times 150^{\prime \prime}$, centered on the IRAS source, to analyze the distribution and characteristics of the molecular gas linked to the IRAS source.

Results. The molecular gas distribution reveals a molecular clump that is coincident with IRAS 08589-4714 and with a dust clump detected at $1.2 \mathrm{~mm}$. The molecular clump is $0.45 \mathrm{pc}$ in radius and its mass and $\mathrm{H}_{2}$ volume density are $310 M_{\odot}$ and $1.2 \times 10^{4} \mathrm{~cm}^{-3}$, respectively. Two overdensities were identified within the clump in $\mathrm{HCN}(3-2)$ and $\mathrm{HCO}^{+}(3-2)$ lines. A comparison of the LTE and virial masses suggests that the clump is collapsing in regions that harbor young stellar objects. An analysis of the molecular lines suggests that they are driving molecular outflows.
\end{abstract}

Key words. ISM: individual objects: IRAS 08589-4714 - stars: formation - stars: massive - ISM: jets and outflows circumstellar matter - ISM: molecules

\section{Introduction}

IRAS 08589-4714 (RA, Dec. $(J 2000)=09: 00: 40.5,-47: 25: 55)$ can be classified as an ultracompact HII region (UCHII) according to the criteria by Wood \& Churchwell (1989). This source coincides with a massive dust clump detected in the IR continuum at $1.2 \mathrm{~mm}$ by Beltrán et al. (2006). They estimated a luminosity of $1.8 \times 10^{3} L_{\odot}$ and a mass of $40 M_{\odot}$ for this object.

Wouterloot \& Brand (1989) detected emission in the ${ }^{12} \mathrm{CO}(1-0)$ molecular line (angular resolution: 43") toward the IRAS source at $V_{\mathrm{LSR}}=+5.2 \mathrm{~km} \mathrm{~s}^{-1}$. The molecular line shows an asymmetry in the blueshifted peak that is likely produced by noncentral self-absorption and a wing extended toward the red, which is a tracer of a potential outflow. Bronfman et al. (1996) observed the source in the $\mathrm{CS}(2-1)$ molecular line at $V_{\mathrm{LSR}}=+4.3 \mathrm{~km} \mathrm{~s}^{-1}$, and Urquhart et al. (2014) detected emission from the high density ammonium molecular tracer. The central velocity coincides with that of the CS line. With velocities in the range $4-5 \mathrm{~km} \mathrm{~s}^{-1}$, the circular galactic rotation model by Brand \& Blitz (1993) predicts a kinematical distance of $2.0 \mathrm{kpc}$. An uncertainty of $0.5 \mathrm{kpc}$ is assumed, adopting a velocity dispersion of $2.5 \mathrm{~km} \mathrm{~s}^{-1}$ for the interstellar molecular gas.

We report molecular line observations of the IRAS source using tracers of low and high density regions with the aim of studying the molecular gas content of the source, identifying dense gas clumps, finding massive young stellar objects (YSOs) linked to the clumps, and identifying possible outflows.

\footnotetext{
* Final reduced APEX data are only available at the CDS via anonymous ftp to cdsarc.u-strasbg. fr (130.79.128.5) or via http://cdsarc.u-strasbg.fr/viz-bin/qcat?J/A+A/594/A115
}

\section{Molecular line observations}

IRAS 08589-4714 was observed with the $12 \mathrm{~m}$ Atacama Pathfinder EXperiment (APEX) telescope ${ }^{1}$, located in Llano de Chajnantor, in the Puna de Atacama, Chile. We carried out observations in the ${ }^{12} \mathrm{CO}(3-2),{ }^{13} \mathrm{CO}(3-2), \mathrm{C}^{18} \mathrm{O}(3-2), \mathrm{HCO}^{+}(3-2)$, and $\mathrm{HCN}(3-2)$ molecular lines using the On-The-Fly mapping technique. These observations were made on 2014 June 16, 19 , and 21 . The $\mathrm{CO}$ isotopes were observed with the receiver APEX-2 in the spectral range of $343.8-347.8 \mathrm{GHz}$ and in the range $328.6-332.6 \mathrm{GHz}$ with a half-power beamwidth of $\sim 18^{\prime \prime}$, while the $\mathrm{HCO}^{+}$and $\mathrm{HCN}$ molecules were observed with the receiver APEX-1 in the spectral range of $265.6-269.5 \mathrm{GHz}$ with an HPBW of 22" (Vassilev et al. 2008).

We mapped an area of $150^{\prime \prime} \times 150^{\prime \prime}$ covering the central region of the IRAS source and the $1.2 \mathrm{~mm}$ emission zone detected by Beltrán et al. (2006). The APEX-1 system temperature is $150 \mathrm{~K}$ and $300 \mathrm{~K}$ for APEX-2. The data reduction was performed according to the standard procedure of the CLASS software, Gildas ${ }^{2}$. The antenna temperature, $T_{\mathrm{A}}$, was transformed to main-beam brightness-temperature $\left(T_{\mathrm{mb}}=T_{\mathrm{A}} / \eta_{\mathrm{mb}}\right)$, using a main beam efficiency $\eta_{\mathrm{mb}}=0.72$ for APEX -1 and APEX-2 (Vassilev et al. 2008). The data were obtained with a velocity resolution of $0.11 \mathrm{~km} \mathrm{~s}^{-1}$. The final rms is $0.3 \mathrm{~K}$.

\footnotetext{
1 Atacama Pathfinder EXperiment. APEX is a collaboration between the Max-Planck-Institut fur Radioastronomie, the European Southern Observatory, and the Onsala Space Observatory.
}

2 http://www.iram. fr/IRAMFR/GILDAS/ 


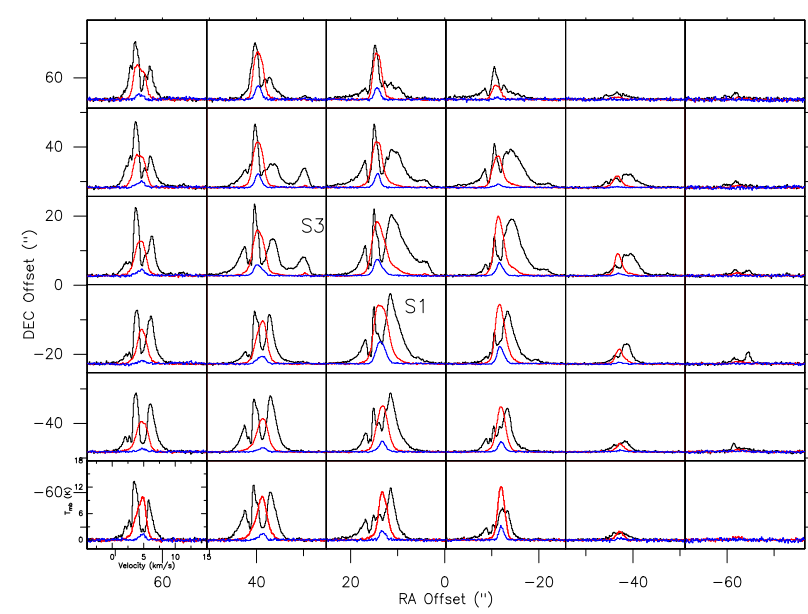

Fig. 1. Spectra of the ${ }^{12} \mathrm{CO}(3-2),{ }^{13} \mathrm{CO}(3-2)$, and $\mathrm{C}^{18} \mathrm{O}(3-2)$ lines indicated in black, red, and blue, respectively. Each spectrum is the average of the profiles within a field of $25^{\prime \prime} .5 \times 25^{\prime \prime} 5$. The total area is $150^{\prime \prime} \times 150^{\prime \prime}$ for three molecules. The locations of sources 1 (S1) and 3 (S3) are labeled. In each panel the velocity ranges from -4 to $+15 \mathrm{~km} \mathrm{~s}^{-1}$ and $T_{\mathrm{mb}}$ scales from -2 to $18 \mathrm{~K}$.

\section{Identification of YSOs}

To investigate the presence of YSOs coincident with the IRAS source in the Wide-field Infrared Survey Explorer (WISE) catalog (Wright et al. 2010), we applied the following criteria (Koenig et al. 2012). Class I objects are those that satisfy $W 1-W 2>1.0$ and $W 2-W 3>2.0$, whereas Class II objects have $W 1-W 2-\sigma_{1}>0.25$ and $W 2-W 3-\sigma_{2}>1.0$, where $W 1$, $W 2, W 3$, and $W 4$ are the magnitudes in the four WISE bands at 3.4, 4.6, 12, and $22 \mu \mathrm{m}$, respectively, and $\sigma_{1}$ and $\sigma_{2}$ are the combined errors of $W 1-W 2$ and $W 2-W 3$, respectively ${ }^{3}$. Three sources with colors of Class I/II were identified. Their coordinates and correlation with 2MASS sources are indicated in Table 1. Sources 1 and 3 are projected onto the dust clump detected by Beltrán et al. (2006).

\section{Molecular line analysis}

\subsection{The ${ }^{12} \mathrm{CO},{ }^{13} \mathrm{CO}$, and $\mathrm{C}^{18} \mathrm{O}$ molecular tracers}

Figure 1 shows the spectra corresponding to the ${ }^{12} \mathrm{CO}(3-2)$, ${ }^{13} \mathrm{CO}(3-2)$, and $\mathrm{C}^{18} \mathrm{O}(3-2)$ molecular lines (indicated in black, red, and blue colors, respectively). In this figure we distinguish two areas in the ${ }^{12} \mathrm{CO}$ molecular emission: for $\Delta \alpha>-40^{\prime \prime}$, the emission is very complex and shows many components with velocities between -3.0 and $+13 \mathrm{~km} \mathrm{~s}^{-1}$, while for $\Delta \alpha<-40^{\prime \prime}$ the emission is weak, below $3 \mathrm{~K}$.

The profiles of the ${ }^{13} \mathrm{CO}(3-2)$ line reveal two distinct regions, as in the case of the ${ }^{12} \mathrm{CO}$ emission. The emission in the ${ }^{13} \mathrm{CO}(3-2)$ spectra has velocities in the range $[+1,+9] \mathrm{km} \mathrm{s}^{-1}$ and only one maximum that is centered between $\sim+4$ and $+5 \mathrm{~km} \mathrm{~s}^{-1}$. The $\mathrm{C}^{18} \mathrm{O}(3-2)$ line profiles are shown in the same area than in the previous cases. The area of more intense emission has a rather elongated shape from NE to SW.

\subsection{Molecular emission toward sources 1 and 3}

Figure 2 shows the spectra of the molecular lines observed toward sources 1 and 3 indicated in Fig. 1. Toward source 1 (see

\footnotetext{
3 http://irsa.ipac.caltech.edu/frontpage/
}

Table 1. Candidate YSOs in the WISE and 2MASS databases detected toward IRAS 08589-4714.

\begin{tabular}{cccc}
\hline \hline Source & WISE source & 2MASS source & Class \\
\hline 1 & J090040.97-472601.1 & $09004142-4726050$ & I \\
2 & J090038.59-472648.5 & $09003861-4726489$ & I \\
3 & J090043.08-472539.5 & $09004309-4725394$ & II \\
\hline
\end{tabular}

the two panels on the left of Fig. 2), the ${ }^{12} \mathrm{CO}(3-2)$ emission consists of three maxima between $\sim 0.0$ and $\sim+12.0 \mathrm{~km} \mathrm{~s}^{-1}$ that are centered at $\sim+2.2,+3.6$, and $+6.3 \mathrm{~km} \mathrm{~s}^{-1}$. The profile shows a strong broadening toward more positive velocities and weaker broadening toward more negative velocities (all velocities are referred to the local standard of rest).

The spectra of the remaining molecules display emission between the two external dashed lines $\left(+2.7<V<+6.3 \mathrm{~km} \mathrm{~s}^{-1}\right)$ with the exception of the ${ }^{13} \mathrm{CO}(3-2)$ and $\mathrm{HCN}(3-2)$ lines, which show weak emission outside this velocity interval. The ${ }^{13} \mathrm{CO}(3-2)$ and $\mathrm{C}^{18} \mathrm{O}(3-2)$ lines show peak temperatures at the same velocity of a depression in the ${ }^{12} \mathrm{CO}(3-2)$ profile, which is a characteristic shared by the $\mathrm{HCO}^{+}(3-2)$ and $\mathrm{HCN}(3-2)$ spectra.

Considering that the emission of $\mathrm{HCO}^{+}(3-2)$ and $\mathrm{HCN}(3-2)$ corresponds to the densest region of the molecular cloud, we adopt $V=+4.6 \mathrm{~km} \mathrm{~s}^{-1}$ as the systemic velocity, $V_{\text {sys }}$, for the molecular counterpart of source 1 (central dashed line in Fig. 2), in agreement with Bronfman et al. (1996).

The second panel on the left of Fig. 2 also shows the spatial distribution of the molecular gas emission in the ${ }^{13} \mathrm{CO}(3-2)$, $\mathrm{C}^{18} \mathrm{O}(3-2), \mathrm{HCO}^{+}(3-2)$, and $\mathrm{HCN}(3-2)$ lines (green contours), integrated within the velocity range enclosed by the two external dashed lines in the first panel. The molecular emission is superposed onto the $4.6 \mu \mathrm{m}$ image (grayscale) and onto Herschel at 500 (a), 350 (b), 250 (c), and $70 \mu \mathrm{m}$ (d) in white contours. The emission of the molecular lines shows a maximum coincident with the brightest region in the far-IR, and the extension of the emission decreases in size from ${ }^{13} \mathrm{CO}(3-2)$ to $\mathrm{HCN}(3-2)$. The dust emission provides evidence for a similar trend from 500 to $70 \mu \mathrm{m}$. The spatial agreement between the line and the continuum emissions reveals the molecular counterpart of the dust clump. The $\mathrm{HCO}+$ and $\mathrm{HCN}$ emission shows a dense molecular region inside a lower density clump depicted by the ${ }^{12} \mathrm{CO}$ and ${ }^{13} \mathrm{CO}$ emissions. The molecular emission covers a region with an equivalent radius of $50^{\prime \prime}$.

Figure 2 also shows the molecular line spectra toward source 3 (see the two panels on the right). The ${ }^{12} \mathrm{CO}(3-2)$ spectrum shows emission between $\sim-2.0$ to $\sim+13.0 \mathrm{~km} \mathrm{~s}^{-1}$ with four peaks. The central velocity of the second peak $\left(\sim+3.6 \mathrm{~km} \mathrm{~s}^{-1}\right)$ is in agreement with the maximum in the remaining molecular spectra $\left(\sim+4.0 \mathrm{~km} \mathrm{~s}^{-1}\right)$, whose emission is enclosed by the two external dashed lines, between +2.7 and $+5.3 \mathrm{~km} \mathrm{~s}^{-1}$.

The spatial distribution of the emission in the ${ }^{12} \mathrm{CO}(3-2)$, ${ }^{13} \mathrm{CO}(3-2), \mathrm{C}^{18} \mathrm{O}(3-2)$, and $\mathrm{HCO}^{+}(3-2)$ lines (green contours) toward source 3 is also shown in Fig. 2, integrated in the range $[+3.0,+4.0] \mathrm{km} \mathrm{s}^{-1}$, and overlaid onto the $4.6 \mu \mathrm{m} \mathrm{im}-$ age (grayscale). This small velocity interval was chosen to highlight the gas linked to source 3 , although the molecular emission linked to this source is detected in the velocity interval $[+2.7,+5.3] \mathrm{km} \mathrm{s}^{-1}$. Both sources 1 and 3 are buried in the same molecular clump. The detection of $\mathrm{HCO}^{+}(3-2)$ toward source 3 also reveals the presence of dense molecular gas, which is linked to this source. 

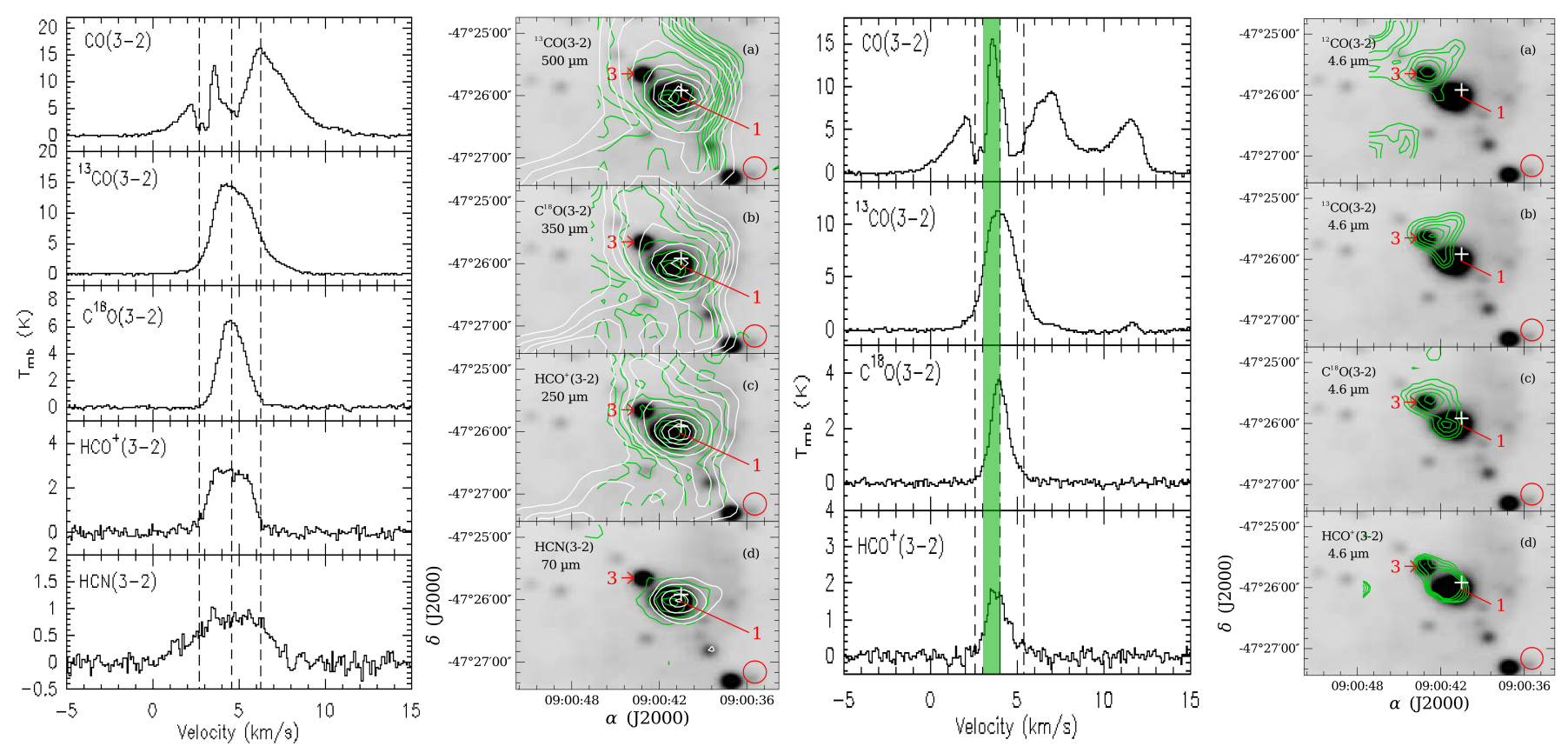

Fig. 2. Left panels: molecular line profiles of ${ }^{12} \mathrm{CO},{ }^{13} \mathrm{CO}, \mathrm{C}^{18} \mathrm{O}, \mathrm{HCO}^{+}$, and $\mathrm{HCN}$ toward source 1 . The vertical dashed central line corresponds to $+4.6 \mathrm{~km} \mathrm{~s}^{-1}$. The two remaining vertical dashed lines indicate the velocity range $[+2.7,+6.3] \mathrm{km} \mathrm{s}^{-1}$. Integrated emission maps for the observed molecules (green contours) superposed over the $4.6 \mu \mathrm{m}$ (grayscale) image. The range of the integrated emission for all molecules is $[+2.7,+6.3] \mathrm{km} \mathrm{s}^{-1}$. The contour levels of the ${ }^{13} \mathrm{CO}$ are between 4 and $47 \mathrm{~K} \mathrm{~km} \mathrm{~s}^{-1}$. The contour levels of $\mathrm{C}^{18} \mathrm{O}$ have values between 1.9 and $13.2 \mathrm{~K} \mathrm{~km} \mathrm{~s}^{-1}$, while the contours of $\mathrm{HCO}^{+}$and $\mathrm{HCN}$ are between $0.8-7.8 \mathrm{~K} \mathrm{~km} \mathrm{~s}^{-1}$ and $1.1-4.1 \mathrm{~K} \mathrm{~km} \mathrm{~s}^{-1}$, respectively. Herschel maps at 500 a), $350 \mathbf{b}), 250 \mathrm{c}$ ), and $70 \mu \mathrm{m} \mathrm{d}$ ) are superimposed in white contours. The white cross indicates the IRAS source position, and the red arrows indicate the location of the identified sources. The HPBW is shown in the lower right corner of each panel. Right panels: molecular line profiles toward source 3. The vertical dashed central line corresponds to $+4.0 \mathrm{~km} \mathrm{~s}^{-1}$. Integrated emission maps of the observed molecules (green contours) superposed onto the $4.6 \mu \mathrm{m}$ (grayscale) image. The integration range for all molecules is $[+3.0,+4.0] \mathrm{km} \mathrm{s}^{-1}$. The velocity range is indicated by the green bar. The contour levels of the $\mathrm{CO}$ are between 6.4 and $8.0 \mathrm{~K} \mathrm{~km} \mathrm{~s}^{-1}$. The contours levels of ${ }^{13} \mathrm{CO}$ have values between 4.5 and $6.5 \mathrm{~K} \mathrm{~km} \mathrm{~s}{ }^{-1}$, while the contours of $\mathrm{C}^{18} \mathrm{O}$ and $\mathrm{HCO}^{+}$are between $1.2-2.3 \mathrm{~K} \mathrm{~km} \mathrm{~s}^{-1}$ and $0.7-1.1 \mathrm{~K} \mathrm{~km} \mathrm{~s}^{-1}$, respectively.

\subsection{Physical parameters of the molecular clump}

From the ${ }^{13} \mathrm{CO}(3-2)$ and $\mathrm{C}^{18} \mathrm{O}(3-2)$ emission lines toward source 1 and 3 , we determine the optical depth of each molecule ( $\tau_{13}$ and $\left.\tau_{18}\right)$, excitation temperature $\left(T_{\mathrm{ex}}\right)$, column density and mass of the molecular clump linked to both sources. The excitation temperature was derived assuming local thermodynamic equilibrium (LTE) conditions and that the emission in the ${ }^{13} \mathrm{CO}(3-2)$ line is optically thick $\left(\tau_{13} \mathrm{CO} \gg 1\right)$. Using the equations by Buckle et al. (2010), the excitation temperature result is $19.3 \mathrm{~K}$. Assuming that $T_{\mathrm{ex}}$ is the same for the two isotopologues, we estimate optical depths for $\mathrm{C}^{18} \mathrm{O}$ and ${ }^{13} \mathrm{CO}$ as 0.6 and 3.2 , respectively.

From the $\mathrm{C}^{18} \mathrm{O}$ column density we estimate the mass as

$M_{\mathrm{H}_{2}}=\left[\mathrm{H}_{2} / \mathrm{C}^{18} \mathrm{O}\right] \mu_{\mathrm{m}} m_{\mathrm{H}} A N\left(\mathrm{C}^{18} \mathrm{O}\right)$,

where $\left[\mathrm{H}_{2} / \mathrm{C}^{18} \mathrm{O}\right]=6 \times 10^{6}$ (Frerking et al. 1982) is the molecular hydrogen-carbon monoxide abundance and $A$ is the area of the clump. From the $\mathrm{C}^{18} \mathrm{O}(3-2)$ contours of Fig. 2, we adopt an equivalent radius of $50^{\prime \prime}$ (or $0.45 \mathrm{pc}$ at $2.0 \mathrm{kpc}$ ) for the molecular clump linked to the sources. Adopting values for $\Delta V_{13}$ and $\Delta V_{18}$ of 2.25 and $1.5 \mathrm{~km} \mathrm{~s}^{-1}$, respectively, we estimate an $\mathrm{H}_{2}$ column density of $1.4 \times 10^{22} \mathrm{~cm}^{-2}$ and a mass of $310 M_{\odot}$. A volume density $n_{\mathrm{H}_{2}} \sim 1.2 \times 10^{4} \mathrm{~cm}^{-3}$ is derived by distributing the molecular mass within a sphere of $0.45 \mathrm{pc}$ in radius. Masses and $\mathrm{H}_{2}$ volume densities are within the values derived for clumps in other regions of the Galaxy.

The detection of $\mathrm{HCO}^{+}$and $\mathrm{HCN}$ toward sources 1 and 3 indicates regions with high ambient densities with values of up to the critical density of the $\mathrm{HCO}^{+}$line $\left(\simeq 3 \times 10^{6} \mathrm{~cm}^{-3}\right)$.

\subsection{Virial mass}

The virial mass of the clump can be obtained from the $\mathrm{C}^{18} \mathrm{O}(3-2)$ line. Considering only gravitational and internal pressure (i.e., neglecting support of magnetic fields, internal heating sources, or external pressure) and assuming a spherically symmetric cloud with an $r^{-2}$ density distribution, the virialized molecular mass of the whole clump, $M_{\text {vir }}$, can be estimated from $M_{\mathrm{vir}}=126 R_{\mathrm{eff}}\left(\Delta v_{\mathrm{cl}}\right)^{2}$ (MacLaren et al. 1988). In this expression, $R_{\mathrm{eff}}=\sqrt{A_{\mathrm{cl}} / \pi}$ is the effective radius in parsecs, $A_{\mathrm{cl}}$ is the area of the clump, and $\Delta v_{\mathrm{cl}}$ is the width of the composite spectrum, which is defined in the same manner as for $\Delta V_{18}$ in Fig. 2. We find that the virial mass is $\sim 135 M_{\odot}$, which is much less than the LTE mass $\left(310 M_{\odot}\right)$; this suggests that the clump is collapsing. This result is compatible with the presence of embedded YSOs.

\section{Molecular outflows}

Figure 3 shows the position-velocity diagrams of the ${ }^{12} \mathrm{CO}(3-2)$ line emission along a cut from the northeast to southwest direction passing through the positions of sources 1 and 3 , where the offset position $0.0^{\prime \prime}$ corresponds to the spectra through the center of source 1 . The brightest emission, with velocities in the range $\sim+2.7<v<+5.0 \mathrm{~km} \mathrm{~s}^{-1}$, within the outer dashed lines in Fig. 2, corresponds to the clump. Figure 3 reveals molecular gas with velocities that are larger than those of the molecular turbulence $\left(\lessgtr+2.5 \mathrm{~km} \mathrm{~s}^{-1}\right)$ outside the mentioned velocity range. The cut shows a prominent redshifted emission from $\sim+5.5$ to $+10 \mathrm{~km} \mathrm{~s}^{-1}$, labeled as $\mathrm{O}-\mathrm{s} 1$, and a less notorious emission from 


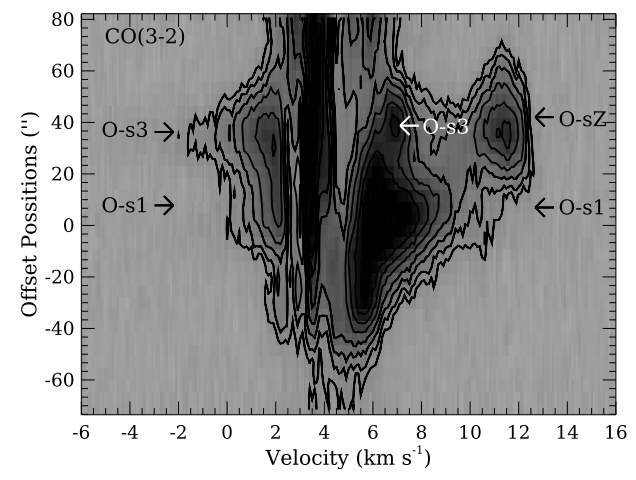

Fig. 3. Position-velocity diagrams of the ${ }^{12} \mathrm{CO}(3-2)$ molecular emission along a line from the northeast to southwest direction through the sources 1 and 3. Contour levels correspond to 1.5, 2.3, 3.5, 5.0, 7.0, 9.0, 11.0, and 14.0 K. Emissions labeled as O-s1 and O-s3 would be the redshifted and blueshifted lobes associated with sources 1 and 3 , respectively.

Table 2. Parameters of the molecular outflows from the ${ }^{12} \mathrm{CO}(3-2)$ line.

\begin{tabular}{lccccc}
\hline \hline Source & Lobe & $\tau_{12}$ & $\begin{array}{c}\phi \\
(\operatorname{arcsec})\end{array}$ & $\begin{array}{c}\Delta v \\
\left(\mathrm{~km} \mathrm{~s}^{-1}\right)\end{array}$ & $\begin{array}{c}M \\
\left(M_{\odot}\right)\end{array}$ \\
\hline 1 & O-s1 blue & - & 30 & 4.5 & 14 \\
& O-s1 red & 18 & 24 & 2.4 & 4.8 \\
\hline 3 & O-s3 blue & - & 33 & 2.5 & 3.8 \\
& O-s3 red & 10 & 25 & 1.8 & 1.7 \\
\hline
\end{tabular}

$\sim+6.0$ to $+8.5 \mathrm{~km} \mathrm{~s}^{-1}$, indicated as $\mathrm{O}-\mathrm{s} 3$ in white. This emission is overlapped by a cloud (O-sZ) showed in the velocity range $\sim+9.5<V<+13.0 \mathrm{~km} \mathrm{~s}^{-1}$. However, this particular emission might also be considered as a small cloud that is extended from $\sim+6.0$ to $+13.0 \mathrm{~km} \mathrm{~s}^{-1}$ and connected to source 3 . For blueshifted velocities, the extended emission is clearly detectable from $\sim-1.5$ to $+2.7 \mathrm{~km} \mathrm{~s}^{-1}$ and from 0.0 to $+2.7 \mathrm{~km} \mathrm{~s}^{-1}$. These emissions would be associated with the redshifted emission O-s3 and O-s1, respectively.

The outflowing gas is generally shown by an optically thick line (such us ${ }^{12} \mathrm{CO}$ ), while the central clump is detected in an optically thin line (such as $\mathrm{C}^{18} \mathrm{O}$ ). Bearing in mind the ${ }^{12} \mathrm{CO}(3-2)$ and $\mathrm{C}^{18} \mathrm{O}(3-2)$ lines (Fig. 2), we take into account that, for source 1 , the blue and red wings are defined in the velocity intervals $[0.0,+2.7]$ and $[+5.5,+10] \mathrm{km} \mathrm{s}^{-1}$, respectively, while for source 3 , these intervals are $[-1.5,+2.7]$ and $[+6.0,+13]$ $\mathrm{km} \mathrm{s}^{-1}$. We believe that molecular material with velocities outside the two dashed lines can be explained as molecular outflows originated in sources 1 and 3 .

In Fig. 4 we show the integrated emission of the $\mathrm{CO}$ line within the redshifted and blueshifted velocity intervals indicated above. Four structures can be distinguished in blueshifted velocities, two of these are over sources 1 and 3 (O-s1 and O-s3) and the other two are labeled as O-sX and O-sY. In redshifted velocities, we find extended molecular emission peaking on sources 1 and toward source 3 . On the contrary, no clear redshifted velocity component is associated with O-sX and/or O-sY. O-sX might be linked to the blueshifted outflows of one of the sources (see Fig. 4), since emission with the velocity range of this component is detected toward both sources in ${ }^{12} \mathrm{CO}$ and ${ }^{13} \mathrm{CO}$.

Adopting an abundance of $\left[{ }^{12} \mathrm{CO} /{ }^{13} \mathrm{CO}\right]=74$ for a galactocentric distance of $8.8 \mathrm{kpc}$ (Wilson \& Rood 1994), we estimate the opacities for the gas in the molecular outflows. Since the blueshifted lobes are not detected at ${ }^{13} \mathrm{CO}(3-2)$, we adopt the same optical depths as for the redshifted lobes. Excitation

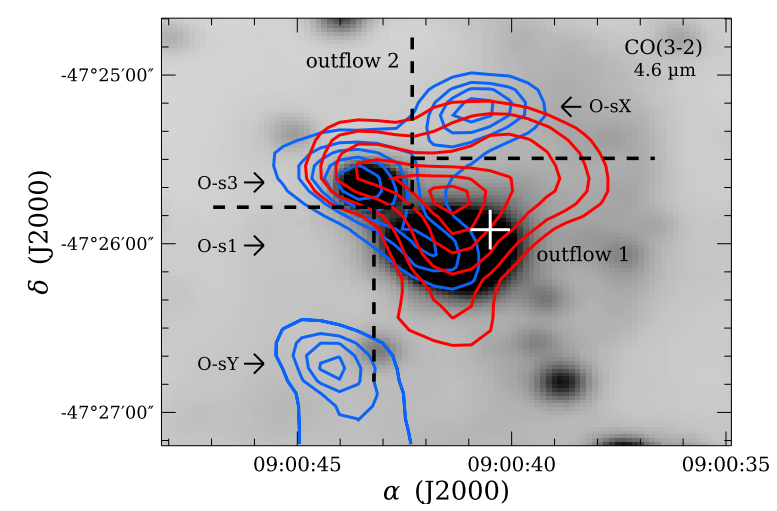

Fig. 4. Integrated map emission of the ${ }^{12} \mathrm{CO}(3-2)$ line wing profiles toward sources 1 and 3 superposed onto the $4.6 \mu \mathrm{m}$ WISE image (grayscale). Light blue contours correspond to 7, 10, 12, 14, and $16 \mathrm{~K} \mathrm{~km} \mathrm{~s}^{-1}$, while red contours correspond to $18,24,32,40,45$, and $48 \mathrm{~K} \mathrm{~km} \mathrm{~s}^{-1}$.

temperatures are $\sim 15 \mathrm{~K}$ for both outflows. Table 2 summarizes the optical depths for redshifted lobes, the effective diameters $\phi$, the velocity width of each lobe, and the masses of the blueshifted and redshifted lobes. The detection of outflows is compatible with previous findings by Wouterloot \& Brand (1989).

\section{Summary}

IRAS 08589-4714 was observed in five molecular lines with the APEX telescope to characterize the molecular environment. An area of $\sim 150^{\prime \prime} \times 150^{\prime \prime}$, centered on the IRAS source position, was covered in the (3-2) transition of ${ }^{12} \mathrm{CO},{ }^{13} \mathrm{CO}, \mathrm{C}^{18} \mathrm{O}$, $\mathrm{HCO}^{+}$, and $\mathrm{HCN}$ lines. A search for candidate YSOs in the WISE database allowed us to identify three IR point sources with characteristics of Class I/II objects according to the criteria by Koenig et al. (2012) within the surveyed region.

The molecular line profiles of ${ }^{12} \mathrm{CO},{ }^{13} \mathrm{CO}$, and $\mathrm{C}^{18} \mathrm{O}$, show multiple velocity components and strong broadening effects toward sources 1 and 3 . The spatial distribution of the $\mathrm{CO}$ emission shows the presence of a molecular clump of $0.45 \mathrm{pc}$ in radius with mass and $\mathrm{H}_{2}$ volume density of $310 M_{\odot}$ and $1.4 \times 10^{4} \mathrm{~cm}^{-3}$, respectively. The comparison between the LTE and virial mass indicates that the clump is collapsing. The $\mathrm{HCO}^{+}$and $\mathrm{HCN}$ spectra reveal molecular overdensities that are coincident with sources 1 and 3. Finally, we detect two possible outflows associated with each source.

Acknowledgements. We thank Ramiro Franco for coordinating the observations with the APEX telescope. H.P.S. acknowledges financial support from a fellowship from CONICET. This project was partially financed by CONICET of Argentina under projects PIP 00356, and PIP 00107 and from UNLP, projects PPID092, PPID/G002, and 11/G120. M.R. wishes to acknowledge support from CONICYT (CHILE) through FONDECYT grant No 1140839.

\section{References}

Beltrán, M. T., Brand, J., Cesaroni, R., et al. 2006, A\&A, 447, 221

Brand, J., \& Blitz, L. 1993, A\&A, 275, 67

Bronfman, L., Nyman, L. A., \& May, J. 1996, A\&AS, 115, 81

Buckle, J. V., Curtis, E. I., Roberts, J. F., et al. 2010, MNRAS, 401, 204

Frerking, M. A., Langer, W. D., \& Wilson, R. W. 1982, ApJ, 262, 590

Koenig, X. P., Leisawitz, D. T., Benford, D. J., et al. 2012, ApJ, 744, 130

MacLaren, I., Richardson, K. M., \& Wolfendale, A. W. 1988, ApJ, 333, 821

Urquhart, J. S., Figura, C. C., Moore, T. J. T., et al. 2014, MNRAS, 437, 1791

Vassilev, V., Meledin, D., Lapkin, I., Belitsky, V., \& et al. 2008, A\&A, 490, 1157

Wilson, T. L., \& Rood, R. 1994, ARA\&A, 32, 191

Wouterloot, J. G. A., \& Brand, J. 1989, A\&AS, 80, 149

Wright, E. L., Eisenhardt, P. R. M., Mainzer, A. K., et al. 2010, AJ, 140, 1868 